\title{
Bioecologia alimentar e reprodutiva como subsídio para o cultivo do peixe Centropomus undecimalis (Teleostei: Centropomidae) no Brasil: Uma Revisão
}

\section{Sistemática}

\author{
Food and Reproductive Bioecology as a subsidy for the cultivation of the fish Centropomus \\ undecimalis (Teleostei: Centropomidae) in Brazil: A Systematic Review \\ Bioecología alimentaria y reproductiva como subvención para el cultivo del pez Centropomus \\ undecimalis (Teleostei: Centropomidae) en Brasil: una revisión sistemática
}

Recebido: 25/11/2021 | Revisado: 04/12/2021 | Aceito: 11/12/2021 | Publicado: 19/12/2021

Isa Rosete Mendes Araújo Nascimento
ORCID: https://orcid.org/0000-0003-0170-765X
Instituto Federal de Educação, Ciência e Tecnologia do Maranhão, Brasil
E-mail: isabio@ifma.edu.br
Jadson Pinheiro Santos
ORCID: https://orcid.org/0000-0002-7521-8835
Universidade Estadual Maranhão, Brasil
E-mail: jadsonsantos@ @rofessor.uema.br
Jéssica Pereira Souza
ORCID: https://orcid.org/0000-0002-6917-8531
Universidade Estadual do Maranhão, Brasil
E-mail: jessicapires1002@ gmail.com
Raimunda Nonata Fortes Carvalho-Neta
ORCID: https://orcid.org/0000-0002-3519-5237
Universidade Estadual Maranhão, Brasil
E-mail: raifortes@ gmal.com
Zafira da Silva Almeida
ORCID: https://orcid.org/0000-0002-8295-5040
Universidade Estadual Maranhão, Brasil
E-mail: zafiraalmeida@ gmail.com

\begin{abstract}
Resumo
Diversos estudos têm sido realizados no Brasil com diferentes enfoques para as avaliações bioecológicas do Centropomus undecimalis (Bloch, 1792), peixe conhecido vulgarmente como robalo ou camurim. Estes estudos descrevem sobre a dinâmica populacional da espécie a partir de dados como peso, idade/crescimento, dieta alimentar e reprodução. Nesse contexto o objetivo do presente trabalho foi realizar uma revisão de literatura sobre a bioecologia alimentar e reprodutiva como subsídio para o cultivo do C. undecimalis no Brasil. A revisão bibliográfica foi realizada de forma sistemática utilizando a biblioteca digital da Scielo (Scientific Electronic Library Online), Google Scholar e ResearchGate, contendo os seguintes descritores em português: "Centropomus undecimalis", "robalo", "camurim"; "robalo-flecha" "bioecologia"; "hábito alimentar", "dieta", "reprodução"; "biologia reprodutiva" e "aspectos reprodutivos", com auxílio dos operadores Booleanos "and", "or" e "and not" e no período compreendido entre 2006 e 2021. Foram identificados 728 artigos relacionados a diferentes aspectos da bioecologia do $C$. undecimalis, dentre eles foram selecionados apenas 24 artigos, identificados como elegíveis para esta revisão sistemática publicados em revistas nacionais e internacionais. Os resultados mostram que o robalo é um peixe carnívoro com tendência piscívora, o que fornece uma ampla compreensão exigência nutricional da espécie para elaboração de rações balanceadas, e que, no Brasil, os estudos que descrevam e caracterizem a reprodução, além dos sistemas de cultivo para os robalos, ainda são escassos.
\end{abstract}

Palavras-chave: Alimentação; Ecologia de peixes; Recurso pesqueiro; Reprodução; Robalo.

\begin{abstract}
Several studies have been carried out in Brazil with different approaches for the bioecological evaluations of Centropomus undecimalis (Bloch, 1792), a fish commonly known as sea bass or chamois. These studies describe the population dynamics of the species from data such as weight, age/growth, diet and reproduction. In this context, the objective of this work was to carry out a literature review on food and reproductive bioecology as a subsidy for the cultivation of $C$. undecimalis in Brazil. The literature review was carried out systematically using the Scielo digital library (Scientific Electronic Library Online), Google Scholar and ResearchGate, containing the following descriptors
\end{abstract}


in Portuguese: "Centropomus undecimalis", "sea bass", "camurim'; "sea bass" "bioecology"; "eating habits", "diet”, "reproduction"; "reproductive biology" and "reproductive aspects", with the help of Boolean operators "and", "or", and "and not" and in the period between 2006 and 2021. A total of 728 articles were identified related to different aspects of the bioecology of $C$. undecimalis, among them, only 24 articles, identified as eligible for this systematic review, published in national and international journals, were selected. The results show that sea bass is a carnivorous fish with a piscivorous tendency, which provides a broad understanding of the nutritional requirement of the species for the preparation of balanced rations, and that, in Brazil, studies that describe and characterize reproduction, in addition to cropping systems for sea bass, they are still scarce.

Keywords: Feeding; Fish ecology; Fishery resource; Reproduction; Common snook.

\section{Resumen}

En Brasil se han realizado varios estudios con diferentes enfoques para las evaluaciones bioecológicas de Centropomus undecimalis (Bloch, 1792), un pez comúnmente conocido como róbalo o lubina. Estos estudios describen la dinámica poblacional de la especie a partir de datos como peso, edad / crecimiento, dieta y reproducción. En este contexto, el objetivo de este trabajo fue realizar una revisión de la literatura sobre bioecología alimentaria y reproductiva como un subsidio para el cultivo de $C$. undecimalis en Brasil. La revisión de la literatura se llevó a cabo sistemáticamente utilizando la biblioteca digital Scielo (Scientific Electronic Library Online), Google Scholar y ResearchGate, que contiene los siguientes descriptores en portugués: "Centropomus undecimalis", "sea bass", "camurim"; "róbalos" "bioecología"; "Hábitos alimenticios", "dieta", "reproducción"; "Biología reproductiva" y "aspectos reproductivos", con la ayuda de operadores booleanos " $y$ ”, " $o$ ” y "y no" y en el período comprendido entre 2006 y 2021. Se identificaron un total de 728 artículos relacionados con diferentes aspectos de la bioecología de $C$. undecimalis, entre ellos, solo se seleccionaron 24 artículos, identificados como elegibles para esta revisión sistemática, publicados en revistas nacionales e internacionales. Los resultados muestran que lo róbalo es un pez carnívoro con tendencia piscívora, lo que proporciona una amplia comprensión del requerimiento nutricional de la especie para la preparación de raciones balanceadas, y que, en Brasil, estudios que describen y caracterizan la reproducción, además de sistemas de cultivo de róbalos, aún son escasos.

Palabras clave: Alimentación; Ecología de peces; Recurso pesquero; Reproducción; Róbalos.

\section{Introdução}

O levantamento de dados e a análise dos aspectos bioecológicos dos peixes aprimoram estudos voltados ao conhecimento da biologia populacional, alimentação, crescimento, reprodução, recrutamento e mortalidade (Vaz-do-Santos et al., 2007; Pereira et al., 2015). Aliados à caracterização dos habitats que compõem o ecossistema, esses estudos se tornam fundamentais aos planos de manejo das espécies bem como para o desenvolvimento de pacotes tecnológicos para o cultivo em cativeiro, em especial o Centropomus undecimalis (Bloch, 1792), que é uma espécie que possui grande valor comercial a partir da exploração pela pesca artesanal, industrial e em pescarias recreativas (Motta et al., 2016).

Conhecer a alimentação natural dos peixes é fundamental para entender melhor de assuntos como a ecologia trófica das comunidades, transferência de energia dentro e entre ecossistemas, sendo uma poderosa ferramenta ecológica para biologia da conservação, a gestão pesqueira (Zavala-Camin, 1996; Lima et al., 2016; Barros, et al., 2021) e a elaboração de rações nutricionalmente balanceadas. Em paralelo, os estudos sobre a biologia reprodutiva nos fornecem dados de grande relevância para que se possa pensar em ordenamentos pesqueiros e gestão adequada da ictiofauna (Almeida et al., 2010), possibilitando ainda a produção de formas jovens em cativeiro.

C. undecimalis é a espécie mais amplamente distribuída do gênero, ocorrendo desde a Carolina do Norte, EUA, Golfo do México e Antilhas, até Santa Catarina, ocasionalmente atingindo o Rio Grande do Sul, Brasil (Figueiredo-Filho et al., 2021). Conhecida popularmente como Camurim (ou Camorim), Robalo-flexa (ou flecha) ou robalo preto e, nos países de língua inglesa, por “common snook” (MPA, 2012), essa espécie apresenta hábito alimentar carnívoro e diversas características que a qualifica para piscicultura, com bom ajuste ao cativeiro e facilidade na aceitação de alimentos inertes com boa taxa de conversão alimentar, e para a pesca esportiva pela voracidade que atacam as iscas, característica marcante em espécies de peixes carnívoros (Alvarez-Lajonchère \& Tsuzuki, 2008; Cerqueira \& Tsuzuki, 2009).

Normalmente, os indivíduos de $C$. undecimalis habitam ambientes estuarinos, e toleram uma ampla variedade de salinidade, o que torna a espécie classificada como eurialina por se aclimatar bem tanto no mar quanto em águas continentais 
(Santos, 2014). E é exatamente essa flexibilidade de adaptação a diferentes variações de salinidade que tem permitido a criação de estratégias para cultivo do robalo que podem favorecer a disponibilidade desse recurso pesqueiro para a comercialização, tendo em vista que se trata de uma espécie de alto valor comercial (Liebl et al., 2016).

Diante da importância do C. undecimelis em toda a zona costeira brasileira objetivou-se, com esta revisão de literatura, levantar e comparar aspectos básicos sobre a bioecologia alimentar e reprodutiva como subsídio ao cultivo do Centropomus undecimalis no Brasil, visto que o conhecimento sobre as características bioecológicas são de suma importância para que se possa estabelecer um ordenamento pesqueiro e uma gestão adequada do recurso, visando garantir a conservação da espécie.

\section{Metodologia}

\section{Estratégia de busca e critérios de elegibilidade}

A revisão bibliográfica foi realizada de forma sistemática utilizando a biblioteca digital da Scielo (Scientific Electronic Library Online), Google Scholar e ResearchGate, contendo os seguintes descritores em português: "Centropomus undecimalis", "robalo", “camurim’; "robalo-flecha" "bioecologia”; "hábito alimentar”, “dieta”, "reprodução”; "biologia reprodutiva" e "aspectos reprodutivos". Para auxiliar a pesquisa, foram utilizados os Operadores Booleanos, "and", "or" e "and not", além do filtro "ano de publicação" delimitando somente os trabalhos publicados entre os anos de 2006 à 2021 para estabelecermos uma análise comparativa criteriosa entre os autores nos últimos 15 anos. Foram identificados 728 artigos relacionados a diferentes aspectos da bioecologia do $C$. undecimalis, dentre eles foram selecionados apenas 24 artigos, identificados como elegíveis para esta revisão sistemática publicados em revistas nacionais e internacionais, excluindo-se teses, dissertações e trabalhos de conclusão de curso bem como aqueles trabalhos que não estavam relacionados com o objetivo do presente estudo. Primeiramente os artigos foram filtrados através da leitura dos títulos, em seguida dos resumos, e após as triagens os artigos que atenderam aos critérios de busca passaram pela etapa de análise de conteúdo com a leitura completa, sendo posteriormente-organizados em tabela em ordem cronológica, contendo os principais tipos de estudo, estado brasileiro onde a pesquisa foi realizada e os principais resultados em cada artigo. Este estudo trata-se de uma pesquisa qualitativa e quantitativa (Ludke, \& Andre, 2013; Estrela, 2018), onde realizamos o levantamento dos artigos em bases de dados e posteriormente procedemos com a identificação, classificação e contagem dos que continham conteúdos relacionados a este trabalho.

\section{Resultados e Discussão}

Na Tabela 1 são mostrados os artigos com as características de cada estudo, abrangendo os autores e ano de publicação, estado do Brasil, tipos de estudos bioecológicos, base de dados e o resultado principal da pesquisa. De modo geral, observou-se um aumento no número de publicações sobre o tema a partir de 2012 representando cerca de 75\% dos artigos analisados, com destaques mais recentes para a possibilidade de produção em cativeiro do robalo visando conservação da espécie. 
Tabela 1. Lista de artigos analisados e selecionados.

\begin{tabular}{|c|c|c|c|c|}
\hline Autor(s)/Ano & Estado & Base de dados & Tipo de Estudo Realizado & Resultado Principal \\
\hline Vaz-dos-Santos, 2007 & SP & Google Scholar & Bioecologia e Gestão da Pesca & $\begin{array}{l}\text { O Brasil carece de uma gestão } \\
\text { pesqueira mais competente. }\end{array}$ \\
\hline Barroso et al., 2007 & $\mathrm{SC}$ & Google Scholar & Alimentação de peixes de cultivo & $\begin{array}{l}\text { Alevinos de Tilápia para peixes de } \\
\text { cultivo }\end{array}$ \\
\hline Anni e Pinheiro, 2009 & $\mathrm{SC}$ & Google Scholar & Hábito Alimentar & $\begin{array}{l}\text { Peixes, crustáceos e insetos } \\
\text { dependendo da tamanho. }\end{array}$ \\
\hline Rabelo et al., 2009 & $\mathrm{PE}$ & Google Scholar & Hábito Alimentar & Peixes, Insetos e Vegetais. \\
\hline Nascimento et al., 2010 & $\mathrm{RN}$ & Google Scholar & Bioecologia & Peixes e Crustáceos \\
\hline Araújo et al., 2011 & $\mathrm{PE}$ & Scielo & Aparato Bucal e Gastrintestinal & $\begin{array}{l}\text { Pequenos copépodas e diferentes } \\
\text { decápodas. }\end{array}$ \\
\hline Nora et al., 2012 & RJ & ResearchGate & Ecologia e Alimentação & Peixes de diferentes espécies. \\
\hline Pereira et al., 2012 & SP & Google Scholar & $\begin{array}{l}\text { Perspectivas para Pisciculturas } \\
\text { Marinhas com robalos }\end{array}$ & $\begin{array}{l}\text { O Brasil tem grande potencial para } \\
\text { piscicultura de espécies marinhas. }\end{array}$ \\
\hline Ferraz et al., 2013 & SC & Google Scholar & Cruzamentos Reprodutivos & $\begin{array}{l}\text { Cruzamento Híbrido em Cultivo de } \\
\text { Peva e Flecha. }\end{array}$ \\
\hline Costa-Filho et al., 2013 & SP & Google Scholar & Cultivo dos robalos & $\begin{array}{l}\text { Destaca a importância do cultivo do } \\
\text { robalo, porém descreve que ainda } \\
\text { há lacunas sobre o conhecimento da } \\
\text { biologia reprodutiva dessa espécie. }\end{array}$ \\
\hline Pereira et al., 2015 & PA & Google Scholar & $\begin{array}{l}\text { Bioecologia alimentar } \\
\text { reprodutiva }\end{array}$ & $\begin{array}{l}\text { Alimentação: Peixes e Crustáceos; } \\
\text { Reprodução: alometria negativa e } \\
\text { fator de condição ideal no mês de } \\
\text { maio. }\end{array}$ \\
\hline $\begin{array}{l}\text { Costa Filho e Mello, } \\
2015\end{array}$ & $\mathrm{CE}$ & Google Scholar & 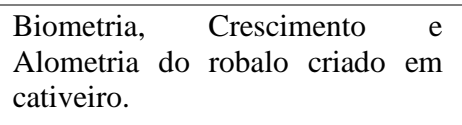 & $\begin{array}{l}\text { Crescimento alométrico positivo e } \\
\text { correlações positivas em juvenis de } \\
\text { robalo-fecha cultivados. }\end{array}$ \\
\hline Herrera et al., 2016 & SP & Scielo & Crescimento e Alimentação & Ingerem Peixes Teleósteos \\
\hline Garcia e Vendel, 2016 & PB & Google Scholar & Sobreposição Alimentar & Peixes, Crustáceos e Poliquetas \\
\hline Farias et al., 2017 & $\mathrm{PE}$ & Google Scholar & Bioecologia Reprodutiva & $\begin{array}{l}\text { Não obteve sucesso com a produção } \\
\text { experimental de híbridos de robalo } \\
\text { peva e flecha }\end{array}$ \\
\hline Lira et al., 2017 & PE & Google Scholar & Ecologia alimentar & $\begin{array}{l}\text { Peixes Teleósteos e Crustáceos } \\
\text { decápodes. }\end{array}$ \\
\hline Pinto et al., 2018 & SE & Google Scholar & $\begin{array}{l}\text { Espacialidades de capturas e } \\
\text { aspectos da biologia dos robalos. }\end{array}$ & $\begin{array}{l}\text { Alometria Positiva para as espécies } \\
\text { estudadas e Fator de condição no } \\
\text { período seco para } C \text {. undecimalis. }\end{array}$ \\
\hline Herrera et al., 2019 & SP & Scielo & Frequência alimentar em cultivo & $\begin{array}{l}\text { Precisam se alimentar a cada } 2 \\
\text { horas }\end{array}$ \\
\hline Teixeira et al., 2019 & SP & Google Scholar & Aspectos Reprodutivos & Período reprodutivo de Nov. a Jan. \\
\hline Souza e Souza, 2019 & AM & Google Scholar & $\begin{array}{l}\text { Produção de alevinos de } C \text {. } \\
\text { undecimalis em cativeiro com alta } \\
\text { salinidade. }\end{array}$ & $\begin{array}{l}\text { Possibilidades de produção em } \\
\text { cativeiro visando conservação da } \\
\text { espécie. }\end{array}$ \\
\hline Silva et al., 2019 & SP & Google Scholar & $\begin{array}{l}\text { Reprodução do robalo segundo } \\
\text { etnoconhecimento de pescadores. }\end{array}$ & $\begin{array}{l}\text { Período reprodutivo } \\
\text { novembro a maio. }\end{array}$ \\
\hline Pereira et al., 2020 & PA & Google Scholar & Bioecologia & Peixes, Crustáceos e Insetos \\
\hline Fonseca et al., 2021 & PI & ReserachGate & Ecologia Alimentar & Carnívoro com dieta variada \\
\hline Souza et al., 2021 & MA & Google Scholar & Ecologia Alimentar & Peixes e Crustáceos \\
\hline
\end{tabular}

Fonte: Autores (2021).

\subsection{Bioecologia alimentar do Centropomus undecimalis no Brasil}

Dentre os 24 artigos descritos na tabela 1, 50\% deles (12 artigos) versavam sobre a bioecologia da alimentação do C. undecimalis, sendo que os demais apresentaram uma mesclagem das discussões a partir de resultados acerca dos aspectos biométricos, reprodutivos e alimentares e de produção em cativeiro. A bioecologia alimentar de uma determinada espécie influencia diretamente na sua dinâmica populacional, sendo indispensável para a conservação dos ecossistemas (Pereira et al., 2015). 
O autor Vaz-dos-Santos, (2007), destaca que a bioecologia é um estudo das integrações obrigatórias entre os aspectos biológicos das espécies e suas relações com o ambiente. As análises realizadas por Rabelo et al. (2009) indicaram os peixes teleósteos como sendo os itens alimentares de maior importância em ocorrência $(69,2 \%)$, seguidos de itens como insetos (7,7\%) e vegetais (7,7\%). Já Anni e Pinheiro (2009) constataram que espécimes de C. undecimalis menores que $35 \mathrm{~cm}$ apresentaram grande parte dos itens alimentares composto por crustáceos além de peixes e insetos, enquanto exemplares maiores que $40 \mathrm{~cm}$ se alimentam preferencialmente de peixes seguido por crustáceos.

Nascimento et al., (2010) realizaram seus estudos com C. undecimalis no Estuário do Potengi, Natal, RN e verificaram que a espécie se apresentou carnívora com tendência a piscívora, revelando uma dieta composta essencialmente por peixes (74\%) e camarões (22\%). Segundo os autores, esses peixes também apresentam grande potencial para criação em cultivo, pois possuem um crescimento satisfatório, adaptação a ambientes salinos, carne de ótima qualidade e boa aceitação no comércio. Nesse sentido, observa-se que o hábito alimentar do peixe é um importante indicador das relações ecológicas entre os organismos, podendo determinar as estratégias de coexistência de espécies afins. Araújo et al. (2011), no intuito de comparar o desenvolvimento do aparato bucal e do trato gastrintestinal de fases iniciais de $C$. undecimalis relacionando-os com a dieta alimentar, identificaram em seus resultados que as larvas $(\mathrm{CP}<10 \mathrm{~mm})$ alimentam-se de pequenos copépodos, enquanto os jovens ( $\mathrm{CP}=11,1$ a $64,7 \mathrm{~mm}$ ) ingerem larvas de diferentes espécies de decápodos, evidenciando uma dieta diferenciada entre as duas fases iniciais de desenvolvimento. Já Nora et al. (2012) encontraram nos estômagos avaliados em juvenis de $C$. undecimalis apenas peixes de diferentes espécies.

Estudos relacionados à bioecologia desse peixe também foram destacados por Pereira et al. (2015), Garcia e Vendel (2016) indicando que de um modo geral, o $C$. undecimalis tem preferência por peixes e crustáceos. Segundo Pereira et al. (2015), robalo-flecha é também uma espécie oportunista pois consome outros alimentos quando há escassez de seus itens preferenciais. Lira et al. (2017) destacaram em seus estudos sobre ecologia alimentar dos Centropomídeos em dois estuários de Pernambuco que a dieta do C. undecimalis em ambas as regiões foi formada por peixes teleósteos e crustáceos - decápodes, neste caso, mostrando-se como carnívoro com tendência piscívora para as duas regiões de estudo.

Pereira et al. (2020) também estudando o camurim no estado do Pará, observaram que ele se alimenta de peixes (81\%), crustáceos (12\%) e Insetos (7\%). Nos estudos de conteúdo alimentar com essa espécie, além dos itens mencionados na presente pesquisa, foi citado também moluscos como categoria alimentar adicional, mas ainda assim houve a preferência por peixes. Estudos feitos por Souza et al. (2021) sobre a Ecologia alimentar do C. undecimalis no Maranhão obtiveram resultados semelhantes sendo $79 \%$ de peixes e $21 \%$ de crustáceos.

Nesse sentido, fica mais evidente a necessidade de se conhecer e compreender as características ecológicas locais, como a disponibilidade de alimentos (Caselle et al. 2011), por conta da particularidade encontrada em cada uma das regiões, sendo de fundamental importância o monitoramento das caraterísticas ecológicas associadas ao ambiente de estudo na tentativa de se evitar sobre-explotação dos estoques pesqueiros e contribuindo de forma mais eficaz na implantação e manutenção de estratégias de gestão dos recursos naturais, mesmo para espécies que se encontram com status de Leas Consern (LC), ou seja, espécie menos preocupante na avaliação feita pela IUCN (2019). De qualquer modo, o estabelecimento de pacotes tecnológicos para o cultivo da espécie depende do conhecimento sobre a bioecologia alimentar, como sugerido por Barroso et al. (2007) após descrever um possível declínio do estoque do C. undecimalis na região do baixo Rio Doce, Espírito Santo.

Percebe-se na tabela 1 que o item peixe foi o alimento preferido pelos robalos nos diferentes estados brasileiros. Nesse contexto, o conhecimento da bioecologia do C. undecimalis torna-se uma estratégia importante para monitorar o status populacional da espécie, auxiliando políticas públicas que visem evitar a depleção dos estoques pesqueiros nas diferentes regiões do Brasil, visto que favorece o manejo adequado da ictiofauna e um ordenamento da pesca do robalo. 


\subsection{Bioecologia Reprodutiva do Centropomus undecimalis no Brasil}

A bioecologia reprodutiva compreende aspectos que são de grande relevância desde o surgimento dos primeiros seres vivos na terra, por permitir o entendimento da importância dos processos reprodutivos como estratégia natural de geração de descentes viáveis a perpetuação das espécies (Vazzoler, 1996; Lowerre-Barbieri et al., 2011). Além disso, os padrões de reprodução mudam de acordo com cada espécie e podem ser favorecidos ou prejudicados de acordo com as características ambientais.

A partir das buscas sistematizadas ficou perceptível que existem poucos trabalhos no Brasil explorando a biologia do Robalo C. undecimalis, contabilizando apenas 33,33\% dos artigos selecionados. Possivelmente, o fato de ser uma espécie de alto valor comercial acaba inibindo a realização de pesquisas por acabar elevando muito o custo para o estudo de sua biologia reprodutiva. A maioria dos trabalhos que envolvem estudos de biologia reprodutiva do $C$. undecimalis foram realizados em regiões e países como: Flórida, México, Venezuela, Colômbia, Costa Rica e Caribe, sendo analisados em ambientes marinhos, dulcícolas ou estuarinos dessas regiões (Taylor et al., 2000; Caballero-Chávez, 2011; Andrade et al., 2013; Gassman et al., 2017); esses autores destacam que o robalo é um peixe hermafrodita protândrico que pode reproduzir o ano todo, pois tem desova fracionada com um pico reprodutivo no mês de junho. Já os estudos feitos no Brasil por Silva et al. (2019) e Nascimento et al. (2021, no prelo) mostram diferença no pico reprodutivo desta espécie.

Teixeira (2019) também realizou trabalhos com as espécies $C$. parallelus, $C$. undecimalis e C. pectinatus com objetivo de analisar aspectos da reprodução dessas espécies com base no conhecimento ecológico local dos pescadores esportivos da Reserva de Desenvolvimento Sustentável da Barra do Una (Peruíbe/SP) e verificou que apenas parte das informações obtidas dos pescadores em relação aos conhecimentos reprodutivos das espécies estudadas foram semelhantes aos da literatura. $\mathrm{O}$ autor observou ainda que os pescadores tiveram mais dúvidas em relação aos peixes maiores como o C. undecimalis, tendo em vista que 55\% dos entrevistados afirmaram não saber distinguir machos e fêmeas pois, segundo eles, é complicado descrever com precisão o sexo deste animal e a única maneira de comprovar essa informação é abrindo o peixe e extraindo as ovas. Apesar das dificuldades relatadas, os pescadores informaram ainda que, normalmente, fêmeas são sempre maiores e mais volumosas que os machos e que o robalo-flecha tem seu período reprodutivo de novembro a janeiro.

Uma outra pesquisa etinobiológica com robalos desenvolvida por Silva et al. (2019) mostrou que a visão dos pescadores amadores em relação ao período reprodutivo do robalo-flecha indica que a espécie reproduz entre os meses de novembro a maio com picos reprodutivos entre janeiro e fevereiro (Figura 1), corroborando com a pesquisa de Nascimento et al. (2021, no prelo), sobre a biologia reprodutiva de C. undecimalis na costa amazônica maranhense. De modo geral, os pescadores amadores e profissionais conseguiram descrever os períodos biológicos das duas espécies de robalo, demonstrando conhecimento ecológico que, em sua maioria, corroborou com a literatura científica. 
Figura 1 - Períodos reprodutivos do Robalo-flecha Centropomus undecimalis segundo a percepção de pescadores amadores em São Paulo.

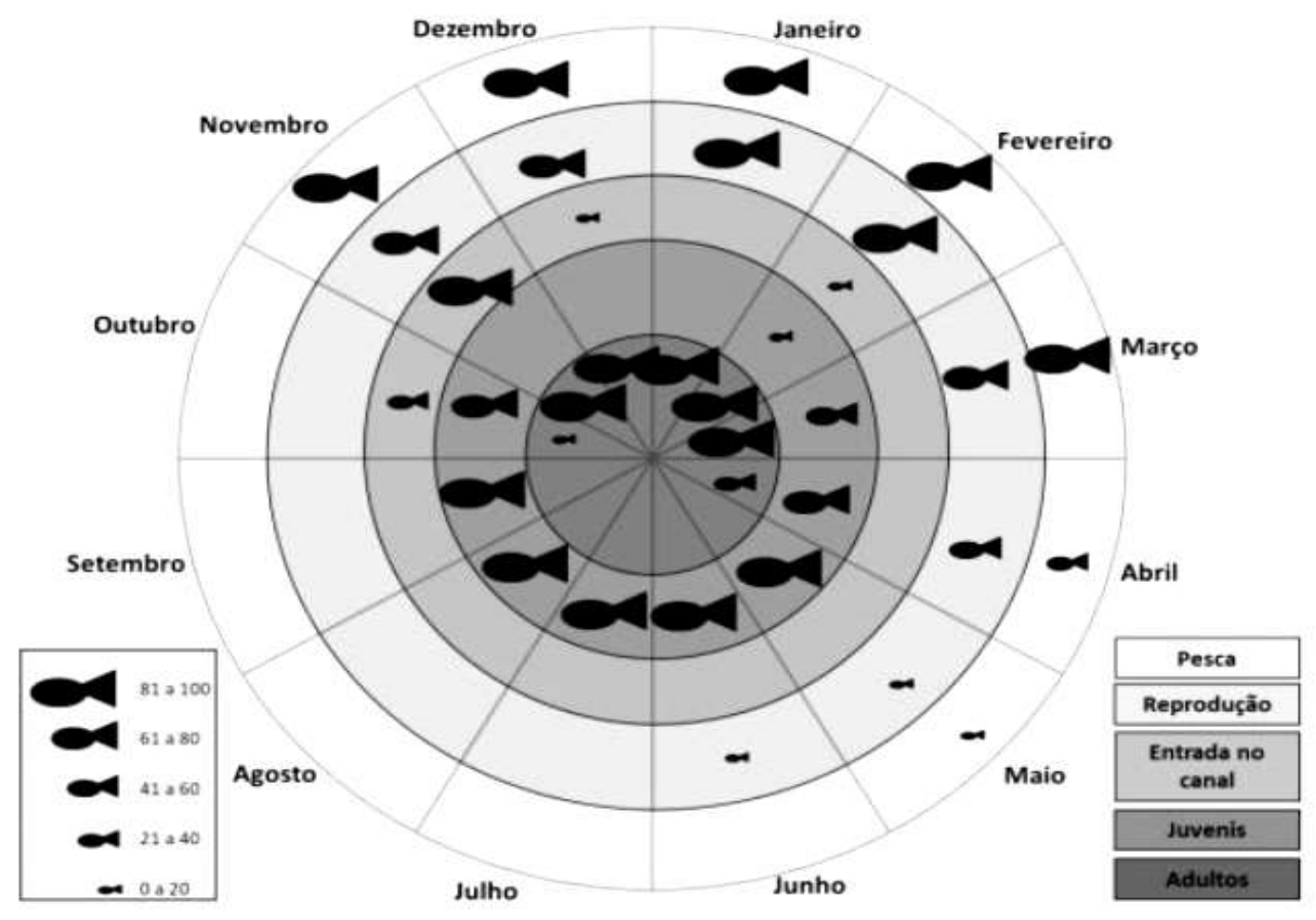

Fonte: Silva et al. (2019).

Pesquisas realizadas por Nascimento et al. (2010) no estuário do Rio Potengi, Rio Grande do Norte, indicaram que a população de robalo-flecha da região era composta na época principalmente por indivíduos machos e jovens imaturos. Foi verificado que, como a espécie apresenta a protandria (ou seja, o indivíduo amadurece primeiro as gônadas masculinas e depois deixa de produzir espermatozoides e amadurece as gônadas femininas), tal fator poderia contribuir mais cedo para o processo reprodutivo e para perpetuação da espécie. Nas populações da Flórida, a transição de macho para fêmea ocorre quando o peixe atinge $51 \mathrm{~cm}$ e 3,4 anos (Taylor et al., 2000). Para o Brasil, essa informação ainda não foi confirmada em nenhum dos estudos realizados até o momento, talvez devido à dificuldade de captura do peixe no momento exato de sua transição.

Estudos feitos por Pereira et al. (2015) na Lagoa Salina, estado do Pará, indicaram que este ambiente tem função de berçário no ciclo de vida do robalo-flecha, revelando uma grande importância enquanto função ecológica e econômica desse ambiente para o recrutamento do $C$. undecimalis na costa norte brasileira, área de grande produção pesqueira. Os autores também identificaram que nessa região a proporção sexual foi assimétrica devido à presença predominante de machos juvenis (imaturos), com destaque para a relação peso-comprimento do robalo-flecha que apresentou alometria do tipo negativa, ou seja, o incremento em comprimento foi inferior ao verificado em peso. Quando avaliados os valores mensais do fator de condição relativo $(\mathrm{Kn})$, observou-se uma variação de 0,8972 a 1,1137, sendo os maiores registrados em maio e os menores em outubro, indicando assim que os robalos-flecha capturados em maio apresentaram melhores condições fisiológicas do que os capturados em outubro.

Vale ressaltar que durante o ciclo de vida dos robalos é preciso um tempo de reclusão geralmente em estuários para ganho de peso e preparação para reprodução (Sousa et al., 2021). Neste sentido, os estudos da bioecologia reprodutiva associada a alimentar deste animal fornecem dados importantes para o seu desenvolvimento de pacotes tecnológicos para o 
cultivo da espécie, pois revelam dados acerca do fator de condição da espécie no ambiente de estudo. Sabendo-se que a reprodução é um processo pelo qual uma espécie se perpetua transmitindo a seus descendentes as mudanças ocorridas em seu genoma, o sucesso obtido por qualquer espécie é determinado, em última instância, pela capacidade de seus integrantes reproduzirem-se em ambientes variáveis mantendo populações viáveis (Vazzoler, 1996; Lowerre-Barbiere et al., 2011), sendo este um fator essencial para a disponibilização de formas jovens de forma eficiente a serem trabalhadas em sistemas de cultivo.

\subsection{A importância da Bioecologia como subsídio para o Cultivo do C. undecimalis no Brasil}

Os conhecimentos bioecológicos sobre o $C$. undecimalis abrem uma possibilidade para elaboração de estratégias que favoreçam a manutenção de seus estoques e o aprimoramento das pesquisas científicas sobre esse recurso. Uma dessas possibilidades é a criação do animal em tanques de cultivo.

Em 2013, Ferraz e colaboradores conduziram experimento com uso do sêmen fresco e criopreservado a partir de tecnologia especialmente desenvolvida para uma tentativa viável de produção de híbridos de Centropomideos, cruzando o robalo-peva (Centropomus parallelus) e o robalo-flecha (Centropomus undecimalis). Porém, os resultados mostraram que houveram baixas taxas de fertilização e sobrevivência das larvas, impossibilitando a obtenção de alevinos dos híbridos pretendidos. Segundo Andrade et al. (2013), a eficiência reprodutiva de diferentes espécies de peixes é dependente de diversos fatores que atuam em conjunto para que a reprodução seja efetiva e produza grande número de larvas sadias. Dentre estes fatores estão o cuidado com o manejo e manutenção dos reprodutores no período pré-reprodução, como alimentação e qualidade da água, até cuidados relacionados com os ovos pós-fertilização, além de cruzamentos interespecíficos viáveis.

Filho e Mello (2015), analisando a biometria, crescimento e alometria do C. undecimalis em ambientes de cultivos, encontraram respostas que indicaram que no ambiente de cultivo estudado os robalos obtiveram correlações positivas e crescimento alométrico positivo. Essas informações são importantes para ampliar o entendimento na avaliação do crescimento dessa espécie em cativeiro. Os dados de Filho e Mello (2015) corroboram com os achados de Pinto et al. (2018) que, realizando um estudo sobre as especialidades de capturas de três espécies de robalos Centropomus parallelus (Poey, 1860), Centropomus undecimalis (Bloch, 1792) e Centropomus pectinatus (Poey, 1860), no estuário do Rio Sergipe, encontraram alometria positiva para todos e maiores valores de $\mathrm{K}$ para o $C$. undecimalis no período seco, o que indica maior armazenamento energético corporal nesse período. Os autores concluíram ainda que este resultado pode ter relação com os eventos reprodutivos desta espécie.-Sobre a possibilidade do cultivo de robalo-flecha em cativeiro como estratégia de conservação da espécie, sabe-se que o $C$. undecimalis é um peixe marinho de grande potencialidade para a piscicultura, devido, principalmente, ao seu alto valor comercial e grande procura no mercado por se tratar de um peixe nobre (Sanches et al., 2014; Liebl et al., 2016). O estado de Santa Catarina foi um dos pioneiros a iniciar o desenvolvimento de pacotes tecnológicos de espécies marinhas, através do cultivo de robalo-flecha (C. undecimalis) e robalo-peva (C. parallelus). Informações sobre reprodução artificial, larvicultura, crescimento e engorda já são de domínio público (Cerqueira, 2004; Pereira et al., 2012).

Além disso, as tecnologias que envolvem a produção de juvenis dessa espécie em laboratório estão razoavelmente bem desenvolvidas. Estudos feitos por Farias et al. (2017) mostram que os juvenis de C. undecimalis apresentaram alta capacidade adaptativa frente à salinidade. $\mathrm{O}$ ciclo de vida dos robalos do gênero Centropomus compreende a reprodução e o início do desenvolvimento larval em áreas costeiras. Os juvenis, por sua vez, são encontrados em manguezais, estuários e lagunas, onde se abrigam e se alimentam, mas também costumam penetrar nos rios e, ocasionalmente, em lagoas hipersalinas.

Souza e Souza (2019) desenvolveram estudos com objetivo de observar o desempenho dos juvenis de C. undecimalis em laboratório criado em águas estuarinas amazônicas, com grande variação de salinidade e ricas em sedimentos. Para os 
indivíduos obtidos através de desova induzida, apresentaram peso médio inicial de $1,3 \mathrm{~g}$ e comprimento total de $4,7 \mathrm{~cm}$, com crescimento dos alevinos no laboratório ao longo dos seis meses acontecendo de forma lenta e heterogênea, o que preliminarmente deixa indícios que na Amazônia o ciclo de cultivo do $C$ - undecimalis é possível, porém mais demorado. Os mesmos autores concluíram ainda que as águas estuarinas do rio Muriá em Curuçá-Pará, foram favoráveis para cultivo de juvenis de robalo-flecha em laboratório, relatando ainda que, mesmo com grande amplitude da salinidade justificada pelos períodos de estiagem e chuva impostos pelo clima amazônico, a taxa de mortalidade foi extremamente baixa dos juvenis de $C$. undecimalis comprovando seu grande poder de adaptação a ambientes com diferentes salinidades. Estudos feitos por Herrera et al. (2019) mostraram que em ambientes de cultivo, os robalos precisam se alimentar em uma frequência de duas em duas horas para garantirem sua sobrevivência.

Percebe-se, portanto, que tanto nos processos reprodutivos naturais, quanto nos artificiais ainda são necessárias mais informações sobre as estratégias reprodutivas do robalo para que se possa garantir a manutenção dos estoques pesqueiros desta espécie em diferentes ambientes e por bastante tempo, mesmo já se tendo descritas algumas tecnologias de cultivo para a espécie, visto que é uma espécie de alto valor comercial e acaba sendo continuamente explorada. Em virtude de a piscicultura ser uma estratégia possível para conservação desta espécie, torna-se de grande necessidade conhecer mais detalhes das taxas de sobrevivência e mortalidade desses indivíduos em viveiros, bem como os pontos chave do ciclo reprodutivo da espécie que favoreçam a maturidade sexual no período adequado e a perpetuação do $C$. undecimalis em todos os ambientes que ele consiga se adaptar.

\section{Conclusão}

De acordo com os dados deste artigo, observa-se que ainda existem lacunas a serem preenchidas acerca dos estudos com a espécie Centropomus undecimalis no Brasil, em especial sobre a reprodução, que gera informações relevantes sobre o desenvolvimento do ciclo gonadal, época e local de desova, comprimento e idade média em que os indivíduos iniciam o processo reprodutivo. Necessita-se também, que os estudos já realizados por pesquisadores sejam publicados em artigos científicos e avaliados por pares, para a divulgação de informações mais precisas sobre a espécie, visto que os estudos bioecológicos de alimentação e reprodução tornam-se subsídios necessários e importantes na regulamentação da pesca, dentro de um programa de manejo, permitindo a tomada de medidas para a preservação dos estoques. Sugere-se então, que novos estudos sejam realizados com robalo-flecha em diferentes classes de comprimento, para descrever melhor a relação com a biologia reprodutiva, alimentação, dinâmica populacional e a biogeografia, a fim de contribuir para a sustentabilidade deste recurso além de subsidiar o desenvolvimento da piscicultura marinha no Brasil como uma estratégia para a conservação da espécie.

\section{Agradecimentos}

Agradeço as professoras Zafira da Silva de Almeida e Raimunda Nonata Fortes Carvalho Neta pela orientação e apoio a esta publicação. Agradeço ainda, aos parceiros Jadson P. Santos e Jéssica P. Souza, pelo auxilio na redação deste artigo e ao Programa de Doutorado em Biodiversidade e Biotecnologia da Rede Bionorte, pelo incentivo a publicação deste artigo.

\section{Referências}

Almeida, Z. S., Isaac Nahum, V. J., Santos, N. B., \& Paz, A. C. (2010) Diagnóstico dos sistemas de produção pesqueiro artesanais do litoral do Maranhão. Ed. UEMA, $126 \mathrm{p}$.

Alvarez-Lajanchère, L. S., \& Tsuzuki, M. Y. (2008) A review of methods for Centropomus spp. (snooks) aquaculture and recommendations for the establishment of their culture in Latin America. Aquaculture Research, 39, 684-700 
Andrade, H., Santos, J., \& Taylor, R. (2013) Life-history traits of the common snook Centropomus undecimalis in a Caribbean estuary and large-scale biogeographic patterns relevant to management. Journal of Fish Biology, 82(6): 1951-1974.

Anni, I. S. A., \& Pinheiro, P. C. (2009). Hábito alimentar das espécies de robalo Centropomus parallelus (Poey, 1986) e Centropomus undecimalis (Bloch, 1792) no litoral norte de Santa Catarina e sul do Paraná, Brasil. In: Anais do III Congresso Latino Americano de Ecologia.

Araújo, I. M. S., Silva-Falcão, E. C., \& Severi, W. (2011). Buccal apparatus and gastrointestinal tract dimensions associated to the diet of early life stages of Centropomus undecimalis (Centropomidae, Actinopterygii) Iheringia, Série Zoologia, 101(1-2):85-92.

Barros, M. F. S., Almeida, Z. S., Figueiredo, M. B., Nunes, J. L. S., \& Carvalho- Neta, R. N. F. (2021) Food ecology of Hassar affinis (Actinopterygii: Doradidae) in two lakes of a wet zone of international importance in Northeast Brazil. Research, Society and Development, 10(8), e10110816973, $10.33448 /$ rsd-v10i8.16973

Barroso, M. V., Souza, G. A. P., Thomé, J. C. A., Leite Júnior, N. de O., Moreira, L. M. P., Sangalia, C., Sales, E. F., \& Durão, JN. (2007). Estratégias de conservação das populações de robalos Centropomus spp. na foz do Rio Doce, Linhares, Espírito Santo, Brasil. I Revista Brasileira de Agroecologia, 2 (2), $1465-1468$.

Brasil/MPA - Ministério da Pesca e Aquicultura. Boletim Estatístico da Pesca e Aquicultura (2012), Brasília. 2011.

Caballero-Chávez V. (2011). Reproducción y fecundidad del robalo blanco (Centropomus undecimalis) en el suroeste de Campeche. Ciencia Pesquera. 19(1):35-45.

Caselle, J. E., Hamilton, S. L., Schroeder, D. M., Milton, S. L., Standish, J. D., Rosales-Casiªn, J. A., \& Sosa-Nishizaki, O. (2011). Geographic variation in density, demography, and life history traits of a harvested, sex-changing, temperate reef fish. Canadian. Journal of Fisheries and Aquatic Sciences, 68(2): 288303.

Cavalli, R. O., \& Hamilton, S. (2007). Piscicultura marinha no Brasil: Afinal, quais as espécies boas para cultivar? Revista Panorama da Aquicultura, 17, 5055 .

Carvalho-Filho, A., Oliveira, J., Soares, C., \& Araripe, J.A. (2019). New species of snook, Centropomus (Teleostei: Centropomidae), from northern South America, with notes on the geographic distribution of other species of the genus. Zootaxa 4671 (1): 081-092. https://doi.org/10.11646/zootaxa.4671.1.6

Cerqueira, V. R., \& Tsuzuki, M. Y. (2009). A review of spawning induction, larviculture, and juvenile rearing of the fat snook, Centropomus parallelus. Fish Physiology and Biochemistry, v. 35, p. 17-28.

Cerqueira, V. R. (2004). Cultivo de peixes marinhos. In: Poli, C. R., Poli, A. T. B., Andreatta, E. R. \& Beltrame, E. (Eds.). Aqüicultura: experiências Brasileiras (pp.369-406). Florianópolis: Multitarefa Editora.

Costa Filho, J., \& Mello, G. L. (2015). Crescimento alométrico positivo entre características biométricas de juvenis de robalo-flecha (Centropomus undecimalis Bloch, 1972) cultivados. Arquivo Ciência Veterinária e Zoologia. UNIPAR, Umuarama, 18(1), 21-26.

Estrela, C. (2018). Metodologia Científica: Ciência, Ensino, Pesquisa. Editora Artes Médicas.

Farias, J. L., Zarar, C. A., Silva-Neto, N. G., Silva, F. S., Silva-Junior, R. F., \& Cavalli, R. O. (2017). Tolerância e crescimento de juvenis do robalo-flecha (Centropomus undecimalis) expostos a diferentes salinidades. Acta Fish 5 (3): 54-60 10.2312/ActaFish.2017.5.3.5460

Ferraz, E. M., Petersen, L., Passini, G., \& Cerqueira, R. (2013). Híbridos Recíprocos Obtidos por Cruzamentos Entre os Robalos Centropomus parallelus e Centropomus undecimalis. Boletim do Instituto de Pesca, 39(1): $53-61$.

Figueiredo-Filho, J. M., Marceniuk, A. P., Feijó, A., Siccha-Ramirez, R., Ribeiro, GS., Oliveira, C., \& Rosa, RS. (2021). Taxonomy of Centropomus Lacépède, 1802 (Perciformes: Centropomidae), with focus on the Atlantic species of the genus. Zootaxa. (3)4942 https://doi.org/10.11646/zootaxa.4942.3.1.

Figueiredo, J. L. \& Menezes, N. A. (1980). Manual de peixes marinhos do sudeste do Brasil: teleostei (2). São Paulo: Museu de Zoologia da USP.

FishBase. Centropomus undecimalis (Bloch, 1792). (2019). https://www.fishbase.se/summary/345

Fricke, R., Eschmeyer, W. N., \& Van der Laan, R. (2019). Eschmeyer's Catalog of fishes: genera, species, references.: http://researcharchive.calacademy.org/research/ichthyology/catalog/fishcatmain.asp .

Fujimoto, R. D., Santana, C. A., Carvalho, W. L. C., Diniz, D. G., Barros, Z. M. N., Varella, J. E. A., \& Guimarães, D. F. (2009) Hematologia e parasitas metazoários de camurim (Centropomus undecimalis, Bloch, 1792) na região Bragantina, Bragança-Pará. Boletim do Instituto de Pesca, 35(3): 441-450.

Garcia, A. F. S., \& Vendel, A. L. (2016). Dietary overlap and food resource partitioning among fish species of a tropical estuary in northeastern Brazil. Gaia Scientia (2016). Volume 10(4): 86-97 http://dx.doi.org/10.21707/gs.v10.n04a06

Gassman J., Rojas H. L.,\& Padrón D. (2017). Reproducción de los robalos Centropomus undecimalis y C. ensiferus (Perciformes: centropomidae) en una laguna costera tropical. Revista de Biología Tropical. 65(1):181-194.

Herrera, L. A., Kuhnen, V. V., \& Sanches, E. G. (2019). Does intensive feeding frequency affect growth performance of common snook Centropomus undecimalis (Bloch, 1792)? Brazilian Journal of Biology https://doi.org/10.1590/1519-6984.186394

IUCN. Mendonça, J. T., Chao, L., Albieri, R. J., Giarrizzo, T., da Silva, F. M. S., Castro, M. G., Brick Peres, M., Villwock de Miranda, L., \& Vieira, J. P. (2019). Centropomus undecimalis. The IUCN Red List of Threatened Species 2019: e. T191835A82665184. http://dx.doi.org/10.2305/IUCN.UK.20192.RLTS.T191835A82665184.

Julião, A. (2019). Unidos pelo robalo. Unespciência, 2018. Edição n.100 Disponível em: http://unespciencia.com.br/2018/09/24robalo100 
Lowerre-Barbieri, S. K., Ganias, K., Saborido-Rey, F., Murua, H., \& Hunter, J. R. (2011). Reproductive timing in marine fishes: variability, temporal scales, and methods. Marine and Coastal Fisheries. 3(1), 71-97.

Liebl, F., Amaral-Junior, H., Garcia, S., Souto, L. I. M., Carvalho, C. V. A., \& Cerqueira, V. R. (2016). Desempenho de juvenis de robalo-flecha e robalo-peva submetidos a diferentes densidades de estocagem em água doce. Boletim do Instituto de Pesca, 42(1): 145-155.

Lima, F. P., Nobile, A. B., Souza-Freitas, D., Carvalho, E. D., \& Vidotto-Magnoni, A. P. (2016). Feeding ecology of Rhinodoras dorbignyi (Kner, 1855) (Siluriformes: Doradidae) in the Paranapanema River, SP, Brazil. Revista Biotemas, 1(29), 67-73.

Lira, A. Z., Frédou, F. L., Viana, A. P., Eduardo, L. N., \& Frédou, T. (2017). Feeding ecology of Centropomus undecimalis (Bloch, 1792) and Centropomus parallelus (Poey, 1860) in two tropical estuaries in Northeastern Brazil. Pan-American Journal of Aquatic Sciences, 12 (2),123-135. Recuperado em 03, Agosto, 2020, de https://panamjas.org/pdf_artigos/PANAMJAS_12(2)_123-135.pdf

Ludke, M. \& Andre, M. E. D. A. (2013). Pesquisas em educação: uma abordagem qualitativa. E.P.U.

Motta, F. S., Mendonça, J. T., \& Moro, P. S. (2016). Collaborative assessment of recreational fishing in a subtropical estuarine system: a case study with fishing guides from south-eastern Brazil. Fisheries Management and Ecology 23, 291-302. https://doi.org/10.1111/fme.12172.

Nascimento, W. S., Gurgel, L. L., Pansard, K. C. A., Nascimento, R. S. S., Gurgel, H. C. B., \& Chellappa, S. (2010). Biologia populacional do robalo-flexa, Centropomus undecimalis (Osteichthyes: Centropomidae) do estuário de rio Potengi, Natal, Rio Grande do Norte, Brasil. Revista Cultural e Científica, 8(3): 65-78.

Nascimento, I. R. M. A., Diniz, A. L. C., Cruz, Y. L. K. F., Azevedo, A. S., Azevedo, R. S. O. P., Pinheiro-Sousa, D. B., Silva, J. C., Carvalho-Neta, R. N. F., \& Almeida, Z. S. (2021) Reproductive biology of the common snook Centropomus undecimalis (Centropomidae: Teleostei) from the northern coast of the Brazilian Amazon. (no Prelo)

Nora, V., Begossi, A., Mesquita, F., Clauzet, M., Rotundo, M. (2012). Aspectos Ecológicos e Etnoecológicos Sobre a Composição Alimentar de Centropomus undecimalis, BLOCH, 1792 (Centropomidae) (robalo) em Paraty, RJ. Unisanta BioScience. 1(1), 22-27.

Pereira, M. E. G. S., Silva, B. B., Rocha, R. M., Asp-Neto, N. E., Silva, C. S., \& Nunes, Z. M. P. (2015). Bioecologia do robalo-flexa, Centropomus undecimalis, em lagoa costeira tropical no norte do BRASIL. Boletim do Instituto de Pesca, 41(3): 457 - 469.

Pereira, M. E. G. S., Cañete, V. R., Palheta, M. K. S., Santos, F. J. S., Silva, B. B., Barbosa, R. S. L., \& Evangelista-Gomes, G. (2020) Contribuição dos atores da pesca para a bioecologia do Camurim Centropomus undecimalis (CENTROPOMIDAE - PERCIFORMES) capturado no litoral Amazônico brasileiro. Research, Society and Development, 9(10), e4119108691, 2020. ISSN 2525-3409 http://dx.doi.org/10.33448/rsd-v9i10.8691

Pinto, J. R. S., Araújo, M. L. G., Rosa, R. A., Santos, J. P., Araújo, A. R. R., \& Barbosa, J. M. (2018). Especialização das capturas de robalo (Centropomus spp) (Centropomidae - Perciformes) no estuário do Rio Sergipe. Arquivos de Ciências do Mar. 51 (2), 72-83.10.32360/acmar.v51i2.33177

Rabelo, L. B., Muto, E. Y., Satiko, L., \& Soares, H. (2009). Observações preliminares sobre o hábito alimentar do robalo-flecha Centropomus undecimalis (BLOCH, 1792) e robalo-peba Centropomus parallelus POEY, 1860, no Estuário de Caravelas (Bahia, Brasil). Boletim Técnico Científico Cepene, (1)17, 8996.

Sanches, E. G., Silva, F. C., \& Ramos, A. P. F. A. (2014). Viabilidade econômica do cultivo do robalo-flecha em empreendimentos de carcinicultura no Nordeste do Brasil. Boletim do Instituto de Pesca, 40(4), 577-588.

Santos, V. L. M. (2014). Biologia reprodutiva e transição sexual do robalo peva: aspectos relevantes para conservação da espécie (Dissertação de mestrado), Universidade Federal do Rio de Janeiro.

Silva, L. M. C., Machado, I. C., Tutui, L. S., \& Tomás A. R. G. (2019) Calendário Etnobiológico do robalo, segundo os pescadores especialistas das áreas estuarinas da Baixada Santista (SP). Investigação qualitativa em ciências sociais. 3. Atas CIAIQ.

Souto, C. N., Faria, T. M., Oliveira, H. F., Rosa, R. M., Silva, L. A. S., \& Campos, M. A. F. (2017). Visão geral sobre reprodução de eixes teleósteos: da anatomia à sinalização molecular. Pubvet, 11(11), 1175-1187.

Souza, J. P., Nascimento, I. R. M. A., Barros, M. F. S., Carvalho, A. S., Brito, P. S., Silva, A. P. C., \& Almeida, Z. S. (2021). Ecologia alimentar do robalo Centropomus undecimalis Bloch 1792 (Teleostei, Centropomidae) na região costeira do Maranhão. Research, Society and Development, $10(9)$, e52010918194. http://dx.doi.org/10.33448/rsd-v10i9.18194

Souza, A. S. L., \& Souza, RAL. (2019). Desenvolvimento de Juvenis de Centropomus undecimalis (BLOCH, 1792) em Laboratório na Amazônia Oriental. Anais do Congresso Brasileiro de Pesca - XXI CONBEP, Manaus -AM.

Teixeira, L. D., Oliveira, P., Souza, U. P., \& Ramires, M. (2019). Aspectos da reprodução dos robalos e o conhecimento ecológico local dos pescadores esportivos da Reserva de Desenvolvimento Sustentável da Barra do Una (Peruíbe/SP). Unisanta, Anais do Encontro Nacional de Pós-Graduação - VIII ENPG Vol.3.

Taylor, R. G., Whittington, J. A., Grier, H. J., \& Crabtree, R. E. (2000). Age, growth, maturation, and protandric sex reversal in the common snook, Centropomus undecimalis, from South Florida waters. Fishery Bulletin, 98(3): 612-624.

Vaz-dos-Santos, A. M., Rossi-Wongtschowski, C. L. D. B., \& Figueiredo, J. L. (2007) Recursos pesqueiros compartilhados: bioecologia, manejo e aspectos aplicados no Brasil. Boletim do Instituto de Pesca, 33(2): 273-292.

Vazzoler, A. E. A. M. (1996) Biologia da reprodução de peixes teleósteos: teoria e prática. SBI/EDUEM. 169p.

Zavala-Camin, L. A. (1996). Introdução aos estudos sobre alimentação natural em peixes. EUDEM, 129p. 